\title{
2D Modeling of Solar Cell p-n Radial Junction: Study of Photocurrent Density and Quantum Efficiency in Static Mode under Monochromatic Illumination
}

\author{
Raguilignaba Sam ${ }^{1,2}$, Alain Diasso², Bernard Zouma ${ }^{3}$, François Zougmoré2 \\ ${ }^{1}$ Département de Physique, UFR/ST, Université Nazi Boni, Bobo Dioulasso, Burkina Faso \\ ${ }^{2}$ Laboratoire de Matériaux et Environnement, Département de Physique, UFR/SEA, Université Joseph Ki-Zerbo, Ouagadougou, \\ Burkina Faso \\ ${ }^{3}$ Laboratoire d'Energies Thermique Renouvelables (L.E.T.R.E), Université Joseph Ki-Zerbo, Ouagadougou, Burkina Faso \\ Email: samrseydou@yahoo.fr, alinodiass@yahoo.fr
}

How to cite this paper: Sam, R., Diasso, A., Zouma, B. and Zougmoré, F. (2020) 2D Modeling of Solar Cell p-n Radial Junction: Study of Photocurrent Density and Quantum Efficiency in Static Mode under Monochromatic Illumination. Smart Grid and Renewable Energy, 11, 191-200.

https://doi.org/10.4236/sgre.2020.1112012

Received: November 14, 2020

Accepted: December 28, 2020

Published: January 25, 2021

Copyright $\odot 2020$ by author(s) and Scientific Research Publishing Inc. This work is licensed under the Creative Commons Attribution International License (CC BY 4.0).

http://creativecommons.org/licenses/by/4.0/

\begin{abstract}
A theoretical study of a polysilicon solar cell with a radial junction in static regime under monochromatic illumination is presented in this paper. The junction radial solar cell geometry is illustrated and described. The carriers' diffusion equation is established and solved under quasi-neutral base assumption with boundaries conditions and Bessel equations. New analytical expressions of electrons and holes photocurrent density and quantum efficiency are found. The wavelength and structural parameters (base radius, base thickness and wavelength) influences on photocurrent density and quantum efficiency are carried out and examined.
\end{abstract}

\section{Keywords}

Grain Geometry, Photocurrent Density, Quantum Efficiency, Radial Junction

\section{Introduction}

The importance that has taken renewable energies in the search for alternative sources of energy to fossil traditional energies because of their rarefaction and harmful impact on the environment is translated specially to photovoltaic energy by many studies on the solar cells. We can obtain energy by converting the solar energy to electrical energy with the semiconductor optoelectronic device such as the vertical junction solar cell or planar junction [1]. Solar cell geometry 
seems to be very important for solar high performance. Especially since study conducted on cubic or cylindrical solar cell [2] showed a slight improvement in solar cell parameters in favor of a cylindrical model notably the radial junction solar cell [3]. The quantum efficiency is a fundamental parameter of the solar cell. Its knowledge allows determining of recombination parameters of solar cell as effective diffusion length, the effective lifetime and recombination velocity because it depends on recombination and generation phenomena in the solar cell. Several studies [4]-[9] have led to find these above parameters in order to characterize the solar cell. The originality of this paper lays its innovative and efficient aspect. The aim of this study is to investigate photocurrent density and quantum efficiency respectively in the base of a radial junction polycrystalline solar cell.

\section{Materials and Methods}

In this study based on a 2D modeling of a polycrystalline silicon radial junction solar cell, we made the following hypothesis:

- The grains have identical structures and electrical properties. Therefore, the study is restricted to a grain whose model is schematized in Figure 1;

- The solar cell is subjected to monochromatic front side illumination;

- The electrical field of crystal lattice and the thickness of space charge region are neglected.

Figure 1 presents a radial junction solar cell geometry:

The operation of radial junction solar cell is based on optical absorption in vertical direction $(z)$. The photogenerated carriers' charge collection is made in radial direction.

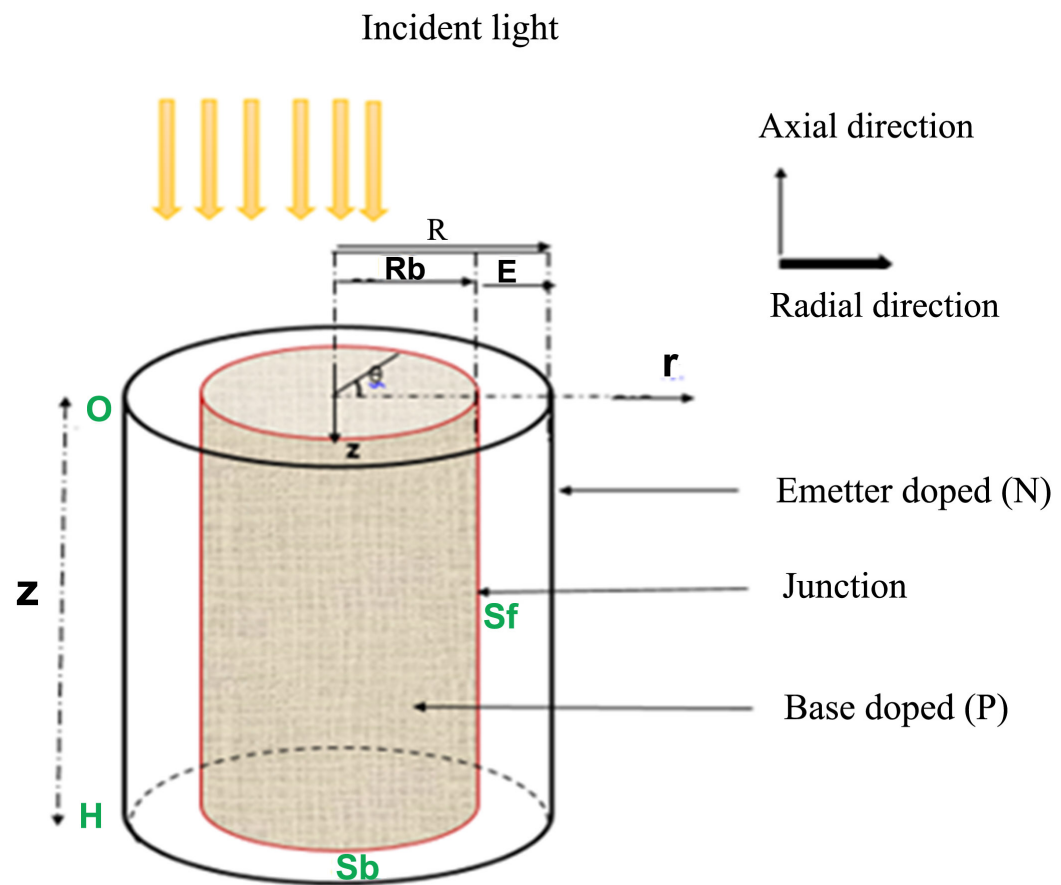

Figure 1. Radial junction model. 


\subsection{Study of Photocurrent Density and Quantum Efficiency}

The transfer phenomenas of solar cell are model by the following equation of continuity numbered Equation (1) in cylindrical coordinates [10].

$$
\begin{aligned}
& \frac{\partial^{2} \delta(r, \theta, z)}{\partial r^{2}}+\frac{1}{r} \frac{\partial \delta(r, \theta, z)}{\partial r}+\frac{1}{r} \frac{\partial^{2} \delta(r, \theta, z)}{\partial \theta^{2}} \\
& +\frac{\partial^{2} \delta(r, \theta, z)}{\partial z^{2}}-\frac{\delta(r, \theta, z)}{L^{2}}=-\frac{g(\lambda, z)}{D}
\end{aligned}
$$

where:

$g(\lambda, z)$ is carriers generation rate [10] [11]:

$$
g(\lambda, z)=\alpha(\lambda) \phi_{0}[1-R(\lambda)] \mathrm{e}^{-\alpha(\lambda) z}
$$

- $\quad \delta(r, z):$ minority carriers' density;

- $D$ : carriers' diffusion coefficient;

- $\quad L$ : carriers' diffusion length.

As, we have an azimuthal symmetry, the angle $\theta$ is not processed [10] [11]. Therefore the continuity equation becomes:

- In the base region, the minority carriers' movement is governed by:

$$
\frac{\partial^{2} \delta_{n}(r, z)}{\partial r^{2}}+\frac{1}{r} \frac{\partial \delta_{n}(r, z)}{\partial r}+\frac{\partial^{2} \delta_{n}(r, z)}{\partial z^{2}}-\frac{\delta_{n}(r, z)}{L_{n}^{2}}=-\frac{g(\lambda, z)}{D_{n}}
$$

\subsubsection{Photocurrent Density Expression in the Base}

Referring to Figure 1, the boundaries conditions are:

- At the junction $\left(r=R_{b}\right)$ :

$$
\left.\frac{\partial \delta_{n}(r, z)}{\partial r}\right|_{r=R_{b}}=-\frac{S_{f}}{2 D_{n}} \delta\left(r=R_{b}, z, \lambda\right)
$$

- At the rear side $(z=H)$ :

$$
\left.\frac{\partial \delta_{n}(r, z)}{\partial z}\right|_{z=H}=-\frac{S_{b}}{D_{n}} \delta(r, z=H, \lambda)
$$

A solution of equation is given by:

$$
\delta_{n}(r, z)=\sum_{k \geq 0}^{\infty} f(r, \lambda) \cos \left(c_{k} z\right)
$$

The eigenvalues $C_{k}$ which satisfy the boundary condition (6) are expressed by:

$$
c_{k} \tan \left(c_{k} H\right)=\frac{\delta_{b}}{D_{n}}
$$

The expression of photocurrent density is given by the following equation:

$$
J_{p h}^{b}=-\left.q D_{n} \int_{0}^{H} \frac{\partial \delta_{n}(r, z)}{\partial r}\right|_{r=R_{b}} \mathrm{~d} z
$$

Substituting $\delta_{n}(r, z)$ in Equation (9); this equation becomes:

$$
J_{p h}^{b}=q \frac{S_{f}}{2 c_{k}} \sum_{k \geq 0}^{\infty}\left(A_{k}(\lambda) I_{0}\left(\frac{R_{b}}{L_{n k}}\right)+M_{k}(\lambda)\right) \sin \left(c_{k} H\right)
$$




\subsubsection{Quantum Efficiency in the Base}

\section{1) Internal quantum efficiency in the base}

The expression of internal quantum efficiency (IQE) is giving by the following expression:

$$
\operatorname{IQE}(\lambda)=\frac{J_{p h}^{B}}{q \phi_{0}[1-R(\lambda)]}
$$

By replacing $J_{p h}^{B} \quad$ expression (10) in Equation (11), we get Equation (12):

$$
\operatorname{IQE}(\lambda)=\sum_{k \geq 0} \frac{-S_{f}}{2 \phi_{0} C_{k}[1-R(\lambda)]} \sin \left(C_{k} \cdot H\right)\left[A_{k}(\lambda) I_{0}\left(\frac{r_{b}}{L_{n k}}\right)+M_{k}(\lambda)\right]
$$

\section{2) External quantum efficiency in the base}

The expression of external quantum efficiency (EQE) is giving by the following expression:

$$
\operatorname{EQE}(\lambda)=\frac{J_{p h}^{B}}{q \phi_{0}}
$$

By replacing $J_{p h}^{B}$ expression (10) in Equation (13), we obtain

$$
\operatorname{EQE}(\lambda)=\sum_{k \geq 0} \frac{-S_{f}}{2 \phi_{0} C_{k}} \sin \left(C_{k} \cdot H\right)\left[A_{k}(\lambda) I_{0}\left(\frac{r_{b}}{L_{n k}}\right)+M_{k}(\lambda)\right]
$$

$I_{0}(x)$ is the modified Bessel function of the first kind of zero order and $I_{0}^{\prime}(x)$ is its first derivative with respect to its argument.

\section{Results and Discussions}

We present here the simulation results obtained from the previous modeling equations by using Mathcad software.

\subsection{Photocurrent Density in the Base}

The effects of wavelength, base radius and base thickness photocurrent density are presented and analyzed.

\subsubsection{Wavelength Effects on Photocurrent Density}

Figure 2 shows the profile of photocurrent density versus wavelength for various values of junction recombination velocity $S_{f}$

We remark a low photocurrent density for low and big wavelength. The absorption peak is notified around $700 \mathrm{~nm}$.

These phenomena are explained by electrons density variations. In additional, photocurrent density increase for $10^{2} \mathrm{~cm} / \mathrm{s} \leq S_{f} \leq 10^{6} \mathrm{~cm} / \mathrm{s}$ because more electrons cross the junction.

For $S_{f} \prec 10^{2} \mathrm{~cm} / \mathrm{s}$, photocurrent density is almost zero because there is a low displacement and strong accumulation of electrons near the junction. Solar cell is then an open circuit state [1] [12].

\subsubsection{Base Radius Effects on Photocurrent Density}

The following figures present variations of photocurrent density as a function 


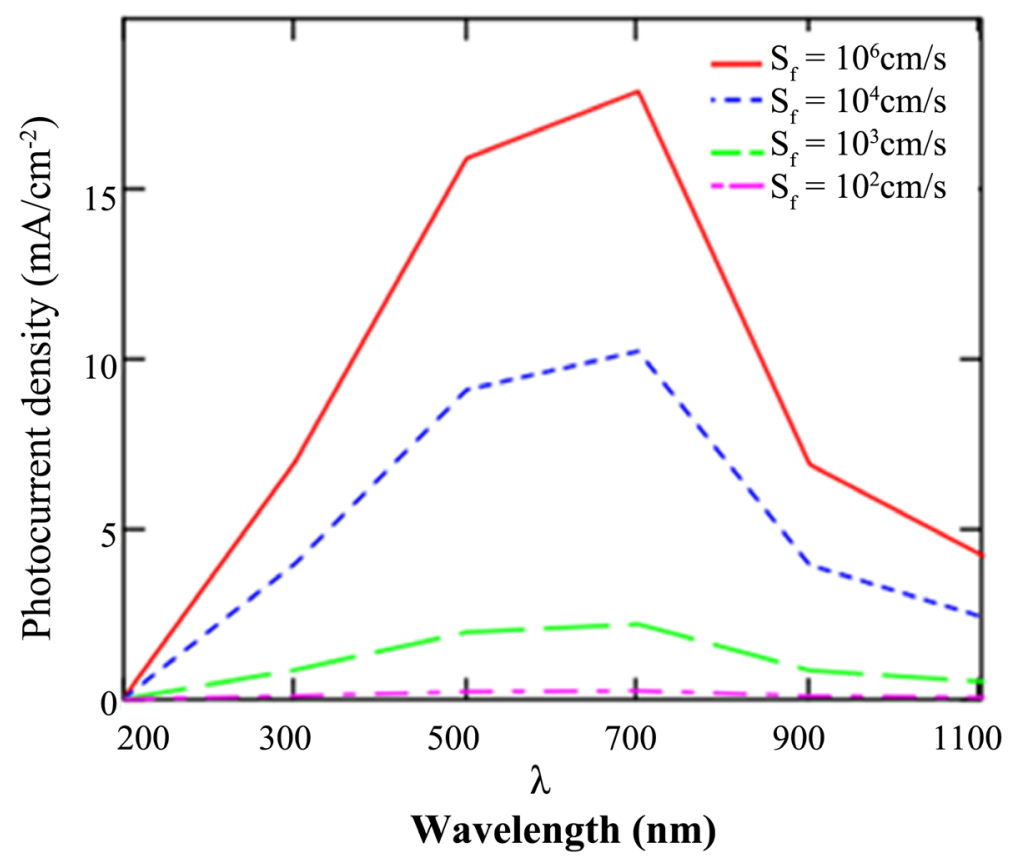

Figure 2. Profile of the photocurrent density as a function of the wavelength for different values of the recombination velocity of the radius $R_{b}$ of the base with, $R_{b}=150 \mu \mathrm{m}, S_{b}=$ $10^{4} \mathrm{~cm} / \mathrm{s}, H=300 \mu \mathrm{m}, L_{n}=120 \mu \mathrm{m}$.

of base radius for different values of the depth creating more electrons because the emitter thickness increase.

On Figure 3, the profile of photocurrent density as a function of dynamic velocity to the junction for various values of base radius $R_{b}$.

The photocurrent increase with the increase of base radius $R_{b}$. The base radius increase creates a large incident light absorption, thus leading to a large of carriers photogenerations in solar cell. Also, this is due to the increasing of base radius minimizes the recombinations which lead to a storage of charge carriers in the base. Results are in good with those obtained by [3] [11] and presented and that lower base radius corresponds to an increase of activities in recombination centers leading to decrease the photocurrent and hence the solar cell efficiency.

\subsection{Internal Quantum Efficiency in the Base}

\subsubsection{Base Radius Effect on Internal Quantum Efficiency}

In Figure 4, we present the IQE as a function of wavelength for different values base radius.

The internal quantum efficiency decrease when the base radius increase. This is a consequence of the increase of carriers' density with base radius because much carriers are collected. Also, the electron maximum decrease in the base depth explaining these variations.

\subsubsection{Base Thickness Effect on Internal Quantum Efficiency}

In Figure 5, we present the variations of internal quantum efficiency as a function of wavelength with various base thickness values. 


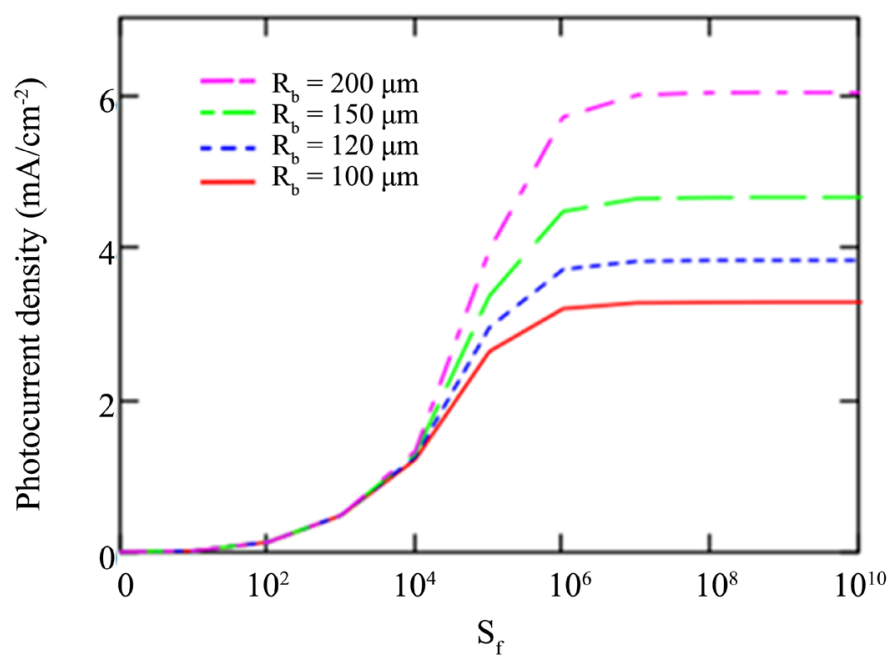

charges carriers' density recombination velocity $(\mathrm{cm} / \mathrm{s})$

Figure 3. Profile of the photocurrent density as a function of the recombination velocity at the junction for different values of the base radius with: $S_{b}=10^{3} \mathrm{~cm} / \mathrm{s}, H=300 \mu \mathrm{m}, L_{n}$ $=120 \mu \mathrm{m}, D_{n}=26 \mathrm{~cm}^{2} / \mathrm{s}$.

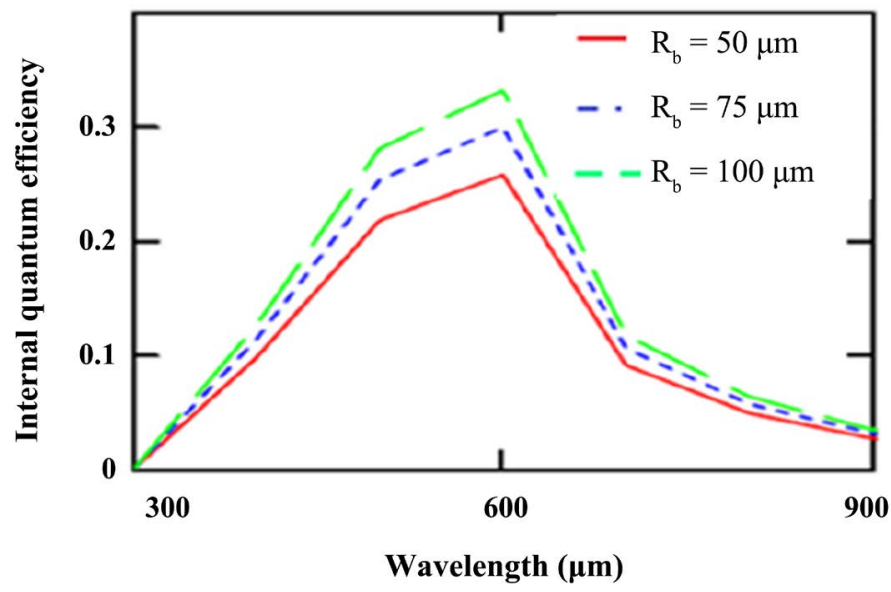

Figure 4. Internal quantum efficiency versus wavelength with various base radius values, $S_{b}=10^{3} \mathrm{~cm} / \mathrm{s}, H=300 \mu \mathrm{m}, L_{n}=120 \mu \mathrm{m}, D_{n}=26 \mathrm{~cm}^{2} / \mathrm{s}$.
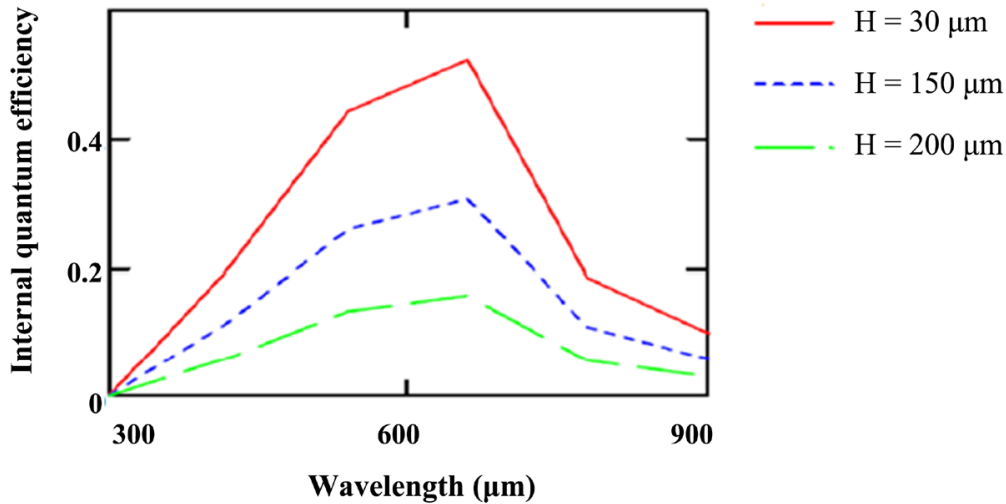

Figure 5. Internal quantum efficiency versus wavelentgth with various base thickness values, $S_{b}=10^{3} \mathrm{~cm} / \mathrm{s}, H=300 \mu \mathrm{m}, L_{n}=120 \mu \mathrm{m}, D_{n}=26 \mathrm{~cm}^{2} / \mathrm{s}$. 
The observing of curves indicate the decrease of internal quantum efficiency with base thickness increase. This increase is relatively greater than the increase with base radius. This increase is explained also by the silicon polycrystalline which grains are small thickness.

\subsubsection{Wavelength Effect on Internal Quantum Efficiency}

In figures, the variations of internal quantum efficiency with wavelength for various values of base thickness and base radius are presented.

Whether Figure 4 or Figure 5, the curves of internal quantum efficiency present three parts:

- $300 \mu \mathrm{m} \leq \lambda \leq 600 \mu \mathrm{m}$, the internal quantum efficiency increase with wavelength for various values of base thickness or base radius because the absorption increase with wavelength up to reach the peak (silicon energy gap 1.12 $\mathrm{eV})$. The generation of electrons is great for small values of wavelength. This low increase is

- The internal quantum efficiency maximum value is reached to $600 \mu \mathrm{m}$. The generation of electron is very important for this wavelength value.

- $600 \mu \mathrm{m} \leq \lambda \leq 900 \mu \mathrm{m}$, the internal quantum efficiency decrease with wavelength for various values of base thickness or base radius. This decrease can be explained by the reduction of electrons density in the base. Also, for big values of wavelength, there is no electron generation in the base.

The different variations of internal quantum efficiency are explained by the photocurrent variations. Also, the internal quantum is as a function of photocurrent.

\subsection{External Quantum Efficiency in the Base}

\subsubsection{Base Radius Effects on External Quantum Efficiency}

The influences of base radius on external quantum efficiency are illustrated in Figure 6.

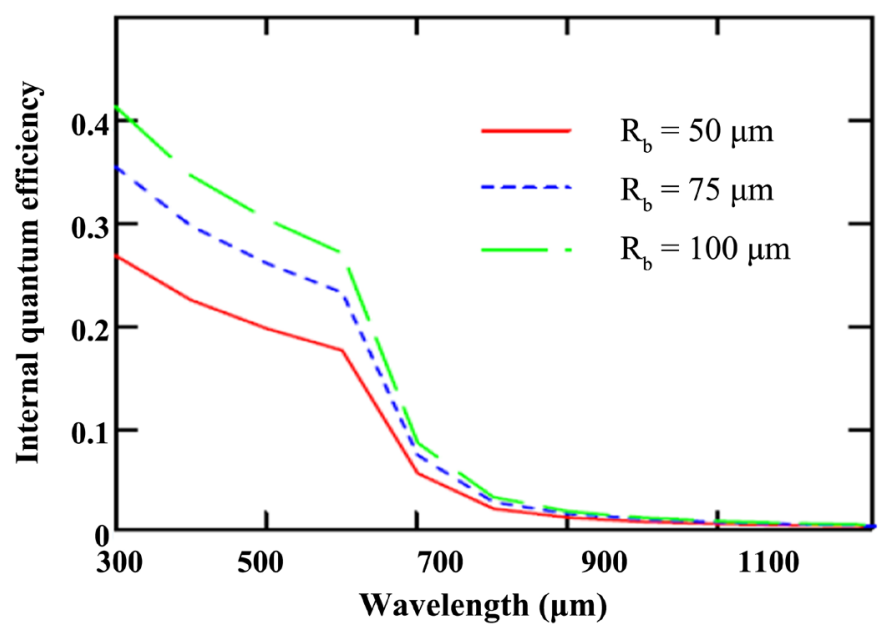

Figure 6. External quantum efficiency versus wavelentgth with various base radius values, $S_{b}=10^{3} \mathrm{~cm} / \mathrm{s}, H=300 \mu \mathrm{m}, L_{n}=120 \mu \mathrm{m}, D_{n}=26 \mathrm{~cm}^{2} / \mathrm{s}$. 


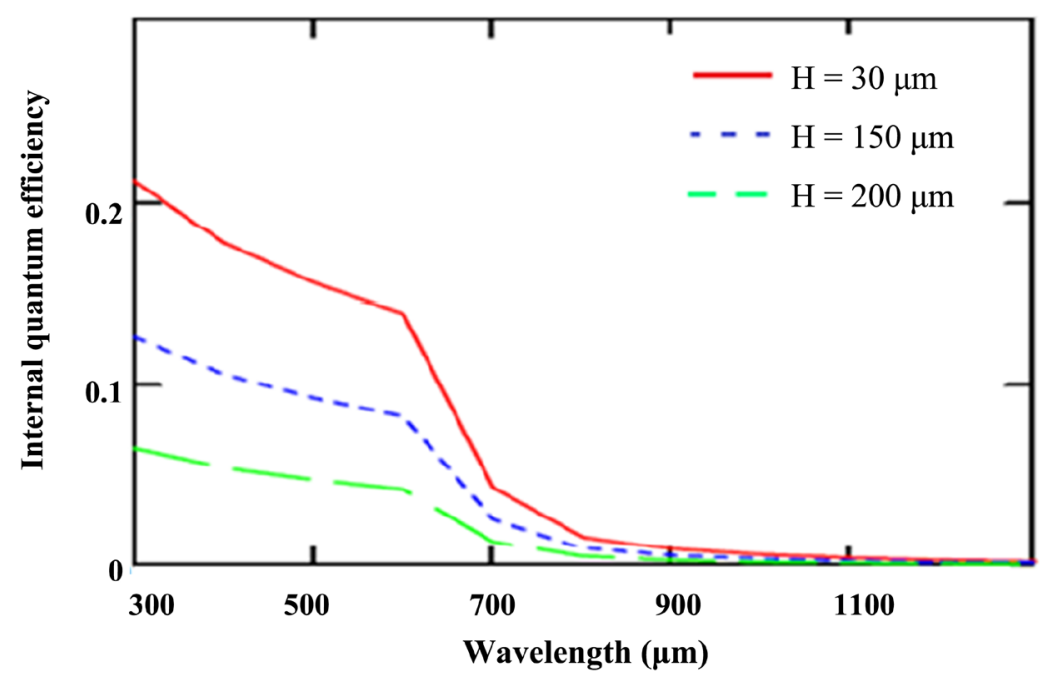

Figure 7. External quantum efficiency versus wavelentgth with various base radius values, $S_{b}=10^{3} \mathrm{~cm} / \mathrm{s}, H=300 \mu \mathrm{m}, L_{n}=120 \mu \mathrm{m}, D_{n}=26 \mathrm{~cm}^{2} / \mathrm{s}$.

The curves analysis indicates that the external quantum efficiency increase crease with base radius. For $\lambda \geq 700 \mu \mathrm{m}$, the decrease is asymptotic as if there is none incident absorption so none photocurrent.

\subsubsection{Base Radius Effects on External Quantum Efficiency}

The impacts of base thickness on external quantum efficiency are illustrated in Figure 7.

The external quantum efficiency decrease with the increase of base thickness. So the reduction of base thickness traduce the decrease of the carriers surface generation.

\subsection{Wavelength Effects on External Quantum Efficiency}

In figures, the curves of external quantum efficiency with wavelength for various values of base thickness and base radius are plotted.

Whether the figure, the curves of internal quantum efficiency present two parts:

- $300 \mu \mathrm{m} \leq \lambda \leq 700 \mu \mathrm{m}$, the decrease of external quantum efficiency is slow.

- $\quad \lambda \geq 700 \mu \mathrm{m}$, the decrease of external quantum efficiency is asymptotic.

For short wavelengths, only a small fraction of the light is converted into electron -holes pairs. Most photons are already absorbed in the layers that the light traverses prior to the absorber layer; this is called parasitic absorption.

\section{Conclusions}

This paper carried out 2D modelling of radial junction of solar cell. The effects of base radius, base thickness and wavelength on carriers' photocurrent density and quantum efficiency are investigated.

Also, the influences of base radius, base thickness and charges carriers' recombination velocity on photocurrent density and quantum efficiency of solar 
cell are presented and analyzed. The results of this study which are in good agreement with other research works [3] [13] validate thus our results.

The $2 \mathrm{D}$ modeling of solar cell $\mathrm{p}-\mathrm{n}$ radial junction quantum efficiency is studied in first time in cylindrical coordinates according to our research. So this work will permit to study better the radial junction of solar cell. In the future, we will try to make an experimental study to confirm theoretical results with experimental results.

\section{Conflicts of Interest}

The authors declare no conflicts of interest regarding the publication of this paper.

\section{References}

[1] Chossat, M. and Privat, Y. (2001) Mathématiques de l'ingénieur Dunod. 4e Edition.

[2] Samb, M.L., Zoungrana, M., Sam, R., Dione, M.M., Deme, M.M. and Sissoko, G. (2010) Etude en modélisation 3D d'une photopile au silicium régime statique placée dans un champ magnétique et sous éclairement multispectral: Détermination des paramètres électriques. Journal des Sciences, N4, 23-38.

[3] Trabelsi, A., Zouri, A. and Arab, A.B. (2009) Modeling of Polycrystalline N+/P Junction Solar Cell with Columnar Cylindrical Grain. Revue des Energies renouvelables, 12, 279-297.

[4] Basu, P.K., et al. (1994) On the Determination of Minority Carrier Diffusion Length in the Base Region of $\mathrm{n}^{+}$-p-p Silicon Solar Cells Using Photoresponse Methods. Solar Energy Materials and Solar Cell, 33, 317-329. https://doi.org/10.1016/0927-0248(94)90234-8

[5] Benmohamed, Z., et al. (2007) Effects of Dislocation Density of Multicrystalline Silicon Solar Cells. Materials and Science Poland, 25, 243-249.

[6] Betser, et al. (1995) Meaurement of the Minority Carrier Mobility in the Base of Heterojunction Bipolar Transistor Using a Magneto Transport Method. Applied Physics Letters, 67, 1883-1894. https://doi.org/10.1063/1.114364

[7] Cuevas, et al. (2002) Millisecond Minority Carrier Lifetime in N-Type Multicrystalline Silicon. Applied Physics Letters, 81, 4952-4954.

https://doi.org/10.1063/1.1529089

[8] Diallo, et al. (2008) New Approach of Both Junction and Back Surface Recombination Velocities in 3D Modelling Study of a Polycrystalline Silicon Solar Cell. The European Physical Journal Applied Physics, 42, 203-211. https://doi.org/10.1051/epjap:2008085

[9] Dieng, A., et al. (2007) Impedance Spectroscopy Methods Applied to Electrical Parameters Determination on Bifacial Silicon Solar Cell under Magnetic Field. Journal des sciences, 7, 48-52.

[10] Elnahwy, S. and Adeeb, N. (1988) Exact Analysis of a Three Dimension Cylindrical Model for a Polycrystalline Solar Cell Department of Engineering Cairo University, Egypt. Journal of Applied Physics, 64, 5214. https://doi.org/10.1063/1.342435

[11] Leye, S.N., Diouf, A., Mbodji, S. and Sissoko, G.G. (2017) A Method to Determine the Transient Capacitance of the Bifacial Solar Cell Considering the Cylindrica Grain and the Dynamic Junction Velocity (Sf). EAI Endorsed Transactions on Collaborative Computing, 3, e5. https://doi.org/10.4108/eai.30-6-2017.153166 
[12] Zouma, B., Maiga, A.S., Dieng, M., Zougmore, F. and Sissoko, G. (2009) 3D Approach of Spectral Response for a Bifacial Silicon Solar Cell under a Constant Magnetic Field. Global Journal of Pure and Applied Sciences, 15, 117-124. https://doi.org/10.4314/gjpas.v15i1.44908

[13] Mbodji, S., et al. (2011) A 3D Model for Thickness and Diffusion Capacitance of Emitter-Base Junction Determination in a Bifacial Polycrystalline Solar Cell under Real Operating Conditions. Turkish Journal of Physic, 35, 281-291. 